\title{
Wireless Sensor Network based Structural Health Monitoring Expert System
}

\author{
B. K Nuhu ${ }^{1}$, O. M. Olaniyi ${ }^{2}$, I. Aliyu ${ }^{3}$, JeKyeong Ryu ${ }^{4}$, C.G Lim 5 \\ 1,2 Department of Computer Engineering, Federal University of Technology, Minna \\ 3,4,5 Department of Computer Engineering, Chonnam National University, Yeosu, \\ South Korea, \\ 1nuhubk@futminna.edu.ng, ${ }^{2}$ mikail.olaniyi@futminna.edu.ng, ${ }^{3}$ Ibal2010@yahoo.com, \\ 41800cc@daum.nets, ${ }^{5}$ cglim@jnu.ac.kr
}

\begin{abstract}
Structural Health Monitoring (SHM) is a process of detecting damages in Engineering structures in order to reduce the frequency of collapse of buildings. In this work, an expert system was incorporated to SHM for monitoring residential buildings. The developed system used a Wireless Sensor Network (WSN) based on 2.4GHz Radio Frequency (RF) band. The developed expert system is based on fuzzy inference and decision-making process also based on British Standard (BS): 7385 - Evaluation and measurement for vibration in building and BS 13670:2009. From performance evaluation result, the system's reliability was calculated to be decreasing from $99 \%$ to $50 \%$ over a space of 100 years. Energy utilization result showed that the system has the capability to operate with $30 \%$ of its energy saved, thereby working for longer hours. The developed system showed a remarkable level of reliability and the energy saving capability is good. Hence, the system is adequate for SHM.
\end{abstract}

Keywords: Structural Health Monitoring, Wireless Sensor Network, Expert System

\section{Introduction}

There has been several incident of collapse of buildings all over the world and this has claimed several lives. Collapse of building can be described as the inability of the building components not to perform what is expected of them [1]. A study showed that 139 houses collapsed between 1974 and 2012 with major occurrences in Lagos and Abuja. One of the noticeable collapses of building is the Lekki Garden building in Lagos which a five storey building under construction collapsed and claimed 30 lives [2]. The causes of collapse of buildings in Nigeria include bad design, faulty construction, use of substandard materials, hasty construction, foundation failure and lack of proper supervision[3].

Structural Health Monitoring (SHM) is a process of detecting damages in Civil Engineering structures. It is a necessary measure that has been identified by the Structural Engineering Community [4]. SHM systems are designed to reliably test and monitor the health performance of structures such as buildings, bridges, sewage and turbines by employing sensors placed at strategic locations where it can give measurement needed in determining the safety of the structures [4][5]. An SHM requires the use of sensors, data transmission and receiving mode,

Article history:

Received (June 26, 2019), Review Result (August 13, 2019), Accepted (October 8, 2019) 
computational power and processing ability. To transmit the structural parameters, recent SHM are configured in Wireless Sensor Network (WSN) mode. WSN is a combination of a number of battery-powered sensor nodes deployed to acquire parameters for monitoring phenomena [6]. Each node consists of different sensor types to monitor different parameters and transmit the data wirelessly between one another to the sink (static or mobile) using any of the existing routing protocols [7][8]. When a mobile sink is employed, the topology changes from time to time and is managed by a topology management protocol[9].

Damage can be considered as the modification of physical parameters such as mass, stiffness and damping. One way through which physical parameters is modified is as a result of motion within the building. This motion can be attributed to vibration within the building. Also, environmental factor such as temperature affects the status of the building.

Expert systems are systems that intelligently take decisions on their own base on the available input and the knowledge programmed in them [10]. They imitate human reasoning about a problem and uses that to solve the problem. Expert systems are implemented using different methods such as Java Expert System Shell (JESS) and Fuzzy Logic. The focus of this paper is to develop a fuzzy logic expert system based SHM using the British Standards (BS: 7385 and BS: 13670)[11], as the standard vibration and temperatures of a building structure respectively. The expert system analyses these two parameters transmitted from the location of measurement via a Zigbee wireless sensor network, to determine the health status of a building. The remaining sections of the paper presents the methodologies of the expert system and WSN designs, the results and discussions, conclusion and future works.

\section{Review of related works}

There exist several SHM systems with most of them employing vibration sensors to ascertain the health of the structures monitored. Some of the works that employed vibration sensors are found in [12][13][14][15] However, each of the works used a different approach in analyzing the vibration data. For instance, in the work of [12], modal parameter (vibration) nondestructive and static tests were used in assessing damages and degree of stiffness degradation. Meanwhile, in the work of [13], their focus was on the sensor and excitation location, environmental effect and effects of different scenarios as it affects the building structures, while in the work of[14], accelerometers and displacement transducers integrated with a temperature sensor were employed to monitor the behavior of cracks on the building. Fibre-optic sensors were also proposed to detect cracks and moisture ingress in a structure [5]. Also, Time Domain Reflectometry (TDR) system incorporated with sensing elements were proposed for monitoring its building heath[16].

\section{Methodology}

The methods adopted for this paper is depicted in Figure 1. It shows the interrelationship between the various components of the system. The system block diagram has two modules: Transmitter modules and the Receiver Module. Using this block diagram, the system can be divided into three sections: data acquisition, data conditioning and transmission, and data reception and processing. 


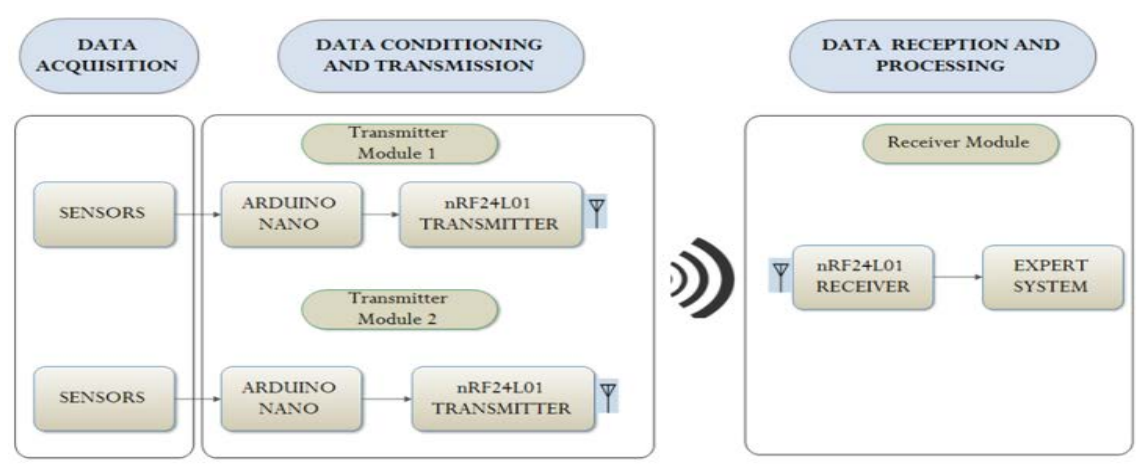

Figure 1. Block diagram of the system

Data Acquisition: The arduino nano is interfaced with the piezo vibration sensor and LM35 sensor. These sensors acquired the vibration and temperature of the building and communicate the data to the arduino nano in which they are connected to.

Data Conditioning and Transmission: At this stage, the data acquired by the sensors is processed by the arduino nano. The processed data is shown on the LCD to ensure accuracy of data at both the transmitting and receiving end. Data is being communicated to the nRF24L01 module from the arduino nano and further transmitted into the network.

Data Reception and Processing: The transmitted data is received at the receiver. The received data from the two transmitters is averaged based on equation 1 and 2 . Then, the average data is rendered to fuzzy logic incorporated into arduino nano for decision making process. The expert system was implemented after the data has been processed.

$$
\begin{gathered}
\text { Temp value }=\frac{t 1+t 2}{2} \\
\text { Vibration value }=\frac{v 1+v 2}{2}
\end{gathered}
$$

Where $t 1=$ Value of temperature sensor $1, t 2=$ Value of temperature sensor $2, v 1=$ Value of vibration sensor $1, v 2=$ Value of vibration sensor 2

\subsection{Design of the Expert System}

The expert system was designed using fuzzy logic. Fuzzy logic is an Artificial Intelligence Technique Capable of making decision. The fuzzy logic system was designed as shown in figure 2. The system has two inputs; temperature and vibration, and an output; status. Figure 2 shows the fuzzy logic system. Fuzzy logic design involved 3 stages: fuzzification, inference engine and defuzzification.

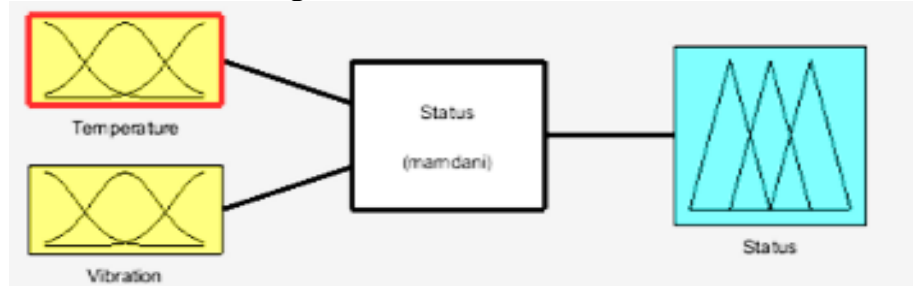

Figure 2. Fuzzy Logic System

\section{Results and Discussion}

\subsection{Energy utilization result}


In order to optimize the energy consumption of the system, the system was designed to work for five minutes and power down the next five minutes in a loop process. A comparison was made between when the system works without power down and power down. This was carried out every five minutes for one hour. The result obtained is shown in Table 1 and Table 2.

The power and energy of the system were calculated using equation 3 and 4 . The percentage change of energy utilized is calculated using equation 5.

$$
\begin{aligned}
& \text { Power, } P=V \times I \\
& \text { Energy, } E=P \times t \\
& \% \text { change of energy utilized }=\frac{E A-E B}{E A} \times 100
\end{aligned}
$$

where $\quad V=$ Voltage, $I=$ Current,$t=$ Time, $E A=$ Energy utilized for Table $1, E B=$ Energy utilized for Table 2

Table 1 is the result of measurement of energy utilized by the system when it worked consistently without sleep mode activation for one hour. Table 2 is the result of measurement of energy utilized by the system when it worked and sleep mode activated every five minutes for one hour. In calculating energy utilized for these tables, percentage change of energy utilized was calculated to be $30 \%$.

Table 1. Result of energy utilization of the system

\begin{tabular}{ccccc}
\hline $\begin{array}{c}\text { Time } \\
(\mathrm{mins})\end{array}$ & $\begin{array}{c}\text { Voltage } \\
(\mathrm{V})\end{array}$ & $\begin{array}{c}\text { Current } \\
(\mathrm{mA})\end{array}$ & $\begin{array}{c}\text { Power } \\
(\mathrm{W})\end{array}$ & $\begin{array}{c}\text { Energy } \\
(\mathrm{J})\end{array}$ \\
\hline 5 & 4.78 & 132 & 0.63 & 0.0525 \\
10 & 4.61 & 133 & 0.61 & 0.1017 \\
15 & 4.81 & 135 & 0.65 & 0.1625 \\
20 & 4.66 & 140 & 0.65 & 0.2167 \\
25 & 4.80 & 142 & 0.68 & 0.2833 \\
30 & 4.69 & 141 & 0.66 & 0.3300 \\
35 & 4.80 & 141 & 0.68 & 0.3967 \\
40 & 4.70 & 141 & 0.66 & 0.4400 \\
45 & 4.82 & 139 & 0.67 & 0.5025 \\
50 & 4.69 & 137 & 0.64 & 0.5333 \\
55 & 4.84 & 137 & 0.66 & 0.6050 \\
60 & 4.69 & 134 & 0.63 & 0.6300 \\
\hline
\end{tabular}

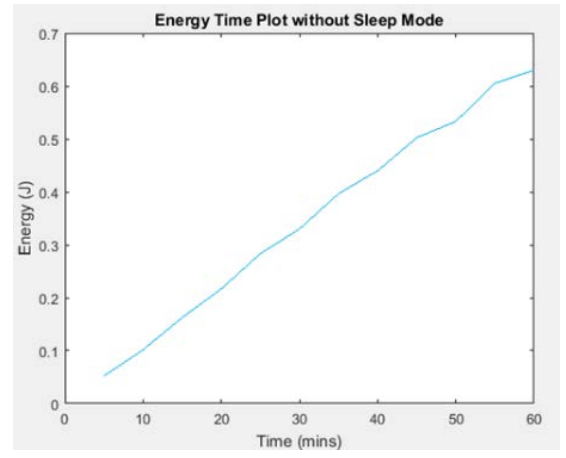

Figure 4: Energy Time Plot without sleep mode

For the result in Table 2, 30\% of energy is saved and this enables the system to work for longer hours. The energy time plot of the system for Table 1 is shown in Figure 4. As the time 
increases, the energy consumed by this system also increases. The energy time plot of the system for Table 2 is shown in Figure 5 . When the sleep mode is activated, energy reduced and increases immediately sleep mode is deactivated.

Table 2. Result of energy utilization of the system

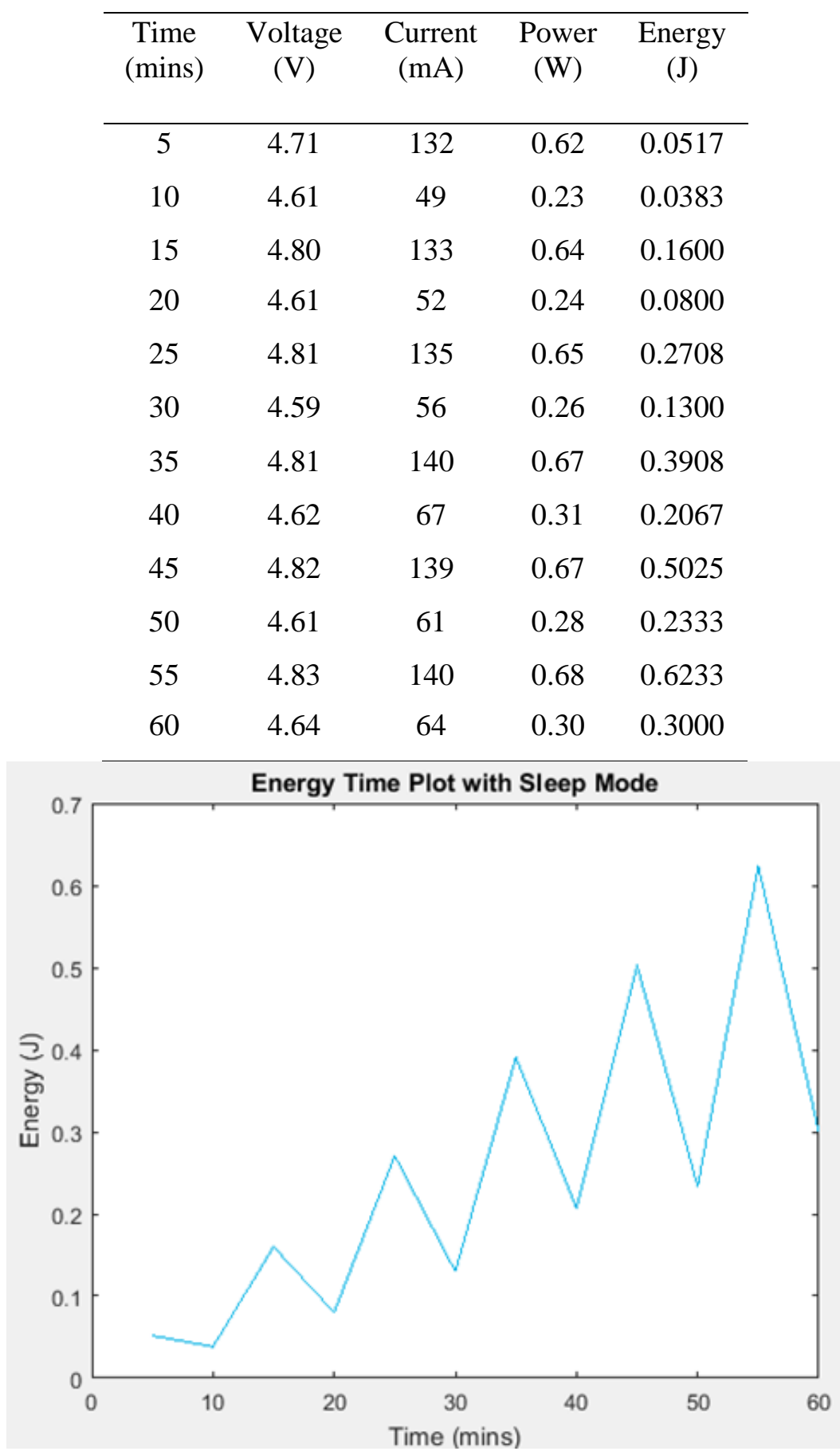

Figure 5. Energy Time Plot with sleep mode 


\subsection{System Reliability Result}

The reliability of the system was calculated (forecasted) in months, and decades. The reliability was forecasted using equation 6 . The forecasted result of the system reliability in months and its graph is shown in Table 3 and Figure 6 (a) respectively. From the table, it is shown that the change in the reliability of the system in months is very small. At some points, the reliability of the system is constant

$$
\text { Reliability, } R_{t}=e^{-\lambda t}
$$

where $\lambda$ =Failure rate, $\quad t=$ Time

Table 3. Reliability of the system in months

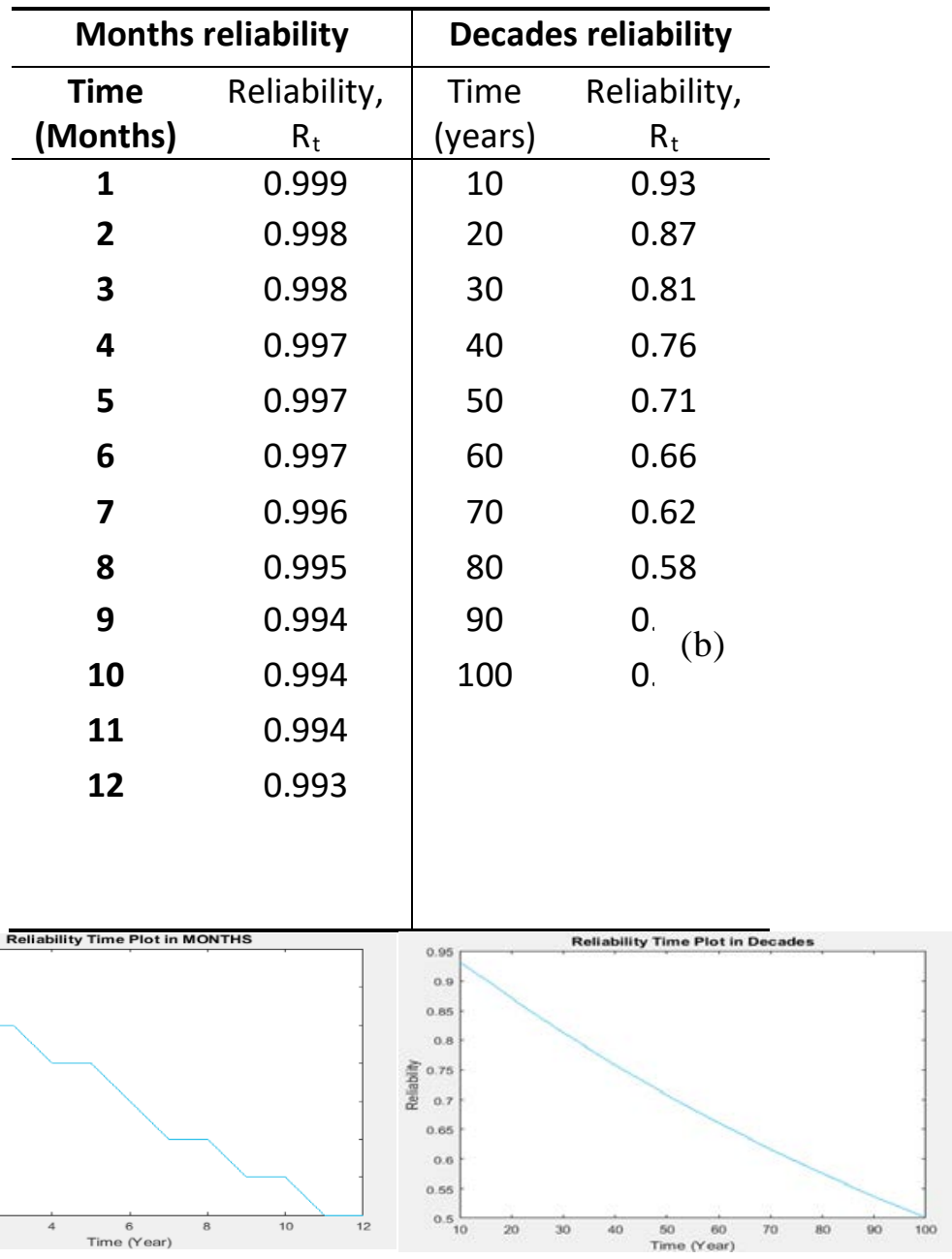

Figure 6. Reliability Time Plot(a) months (b)decades

The result of the calculation of the system reliability in decades and its graph is shown in Table 3 and Figure 6(b) respectively. From the table, it is shown that the reliability of the system decreases as the year increases linearly. No decade has the same reliability. This follows the true curve of life 


\section{Conclusion and Future Works}

The system's reliability was calculated to be decreasing from $99 \%$ to $50 \%$ over a space of 100 years. Energy utilization result showed that the system has the capability to operate with $30 \%$ of its energy saved, thereby working for longer hours. The developed system showed a remarkable level of reliability and the energy saving capability is good. Hence, the system is adequate for Structural Health Monitoring. It is highly recommended that the system should be integrated into buildings especially residential buildings so as to enable the residents to know the status of their buildings at all time. This paper focused on the monitoring of residential structures only, future work would extend it to industrial structures. Also, improvement can further be made to reduce the energy utilization the system.

\section{References}

[1] O. I. Fagbenle, and A. O. Oluwunmi, "Building Failure and Collapse in Nigeria: the Influence of the Informal Sector”, Journal of Sustainable Development, 3(4), pp. 268-276, (2010).

[2] I. Thompson, "Big story taming the spate of building collapse in Nigeria Message posted to Channels TV", Retrieved from: https://www.channelstv.com/?s=big+story+taming+the+spate+of+building+collapse+in+Nigeria, (2016).

[3] E. Badejo, “Engineers; Others Urge Multi-Disciplinary Approach to curb Building Collapse”, The Guardian Newspaper, 13th July, pp. 15-17, (2009).

[4] J. P. Lynch, K. H. Law, A. S. Kiremidjian, T. W. Kenny, E. Carryer and A. Partridge, “The Design of a Wireless Sensing Unit for Structural Health Monitoring”, Proceeding of 3rd International Workshop on Structural Health Monitoring. Stanford, CA, USA, 2001, pp. 1-10, (2001).

[5] K. Bremera, M. Wollweber, F. Weigand, M. Rahlves, M. Kuhne, R, Helbig and B. Roth, "Fibre Optic Sensors for the Structural Health Monitoring of Building Structures”, Proceeding of 3rd International Conference on System-integrated Intelligence: New Challenges for Product and. Elsevier Ltd, 26(2016), pp 524-529, (2016).

[6] Jin Wang, Zhongqi Zhang, Bin Li, Sungyoung Lee, and R. Simon Sherratt, An Enhanced Fall Detection System for Elderly Person Monitoring using Consumer Home Networks, IEEE Transactions on Consumer Electronics, Vol.60, No.1, pp.23-29, Feb. (2014).

[7] Jin Wang, Jiayi Cao, Sai Ji, Jong Hyuk Park, Energy Efficient Cluster-based Dynamic Routes Adjustment Approach for Wireless Sensor Networks with Mobile Sinks, Journal of Supercomputing, Vol.73, No.7, pp.3277-3290, July (2017). DOI: 10.1007/s11227-016-1947-9

[8] Jin Wang, Yu Gao, Wei Liu, Arun Kumar Sangaiah, Hye-Jin Kim, An Intelligent Data Gathering Schema with Data Fusion Supported for Mobile Sink in WSNs, International Journal of Distributed Sensor Networks, vol.15, no.3, Mar. (2019).

[9] Zhang, Wei; Xiong, Naixue; Yang, Laurence T.; Jia, Gangmin; Zhang, Jilin.: BCHED - Energy Balanced SubRound Local Topology Management for Wireless Sensor Network. JOURNAL OF INTERNET TECHNOLOGY, (2012), 13(3): 385-394.

[10] Angeli, Advanced Knowledge Based Systems: Model, Application and Research, TMRF e-book, Vol.1, pp 5073, (2010).

[11] BSI "BS EN 13670:2009: Execution of concrete structures. London, England”, Retrieved from:https://www.construct.org.uk/wpcontent/uploads/2017/01/NSCS-Edition-4 (2009)

[12] N.E. Anthony, U.N. Ben and O.B. Gideon, "Vibration-Based Structural Health Monitoing: Theoretical Foundation and Experimental Validation on Reinforced Concrete Beams”, The International Journal of Engineering and Science, 22(2), pp 1-12, (2015).

[13] A. and E. Anders, "Vibration Health Monitoring for Tensegrity Structures”, Mechanical Systems and Signal Processing, 87(2017), pp 625-637, (2016).DOI: 10.1016/j.ymssp.2016.08.039 
[14] L. Filippo, C. Filippo, C. Mauro, I. Kleidi and M. Claudio, "Uncertainty Quantification in Structure Health Monitoring: Applications on Cultural Heritage Buildings”, Mechanical Systems and Signal Processing, 67(2016), pp 268-281,(2015). DOI: 10.1016/j.ymssp.2015.04.032

[15] L. Xiaoou, Wen, Y., \& Salvador, V. (2015). Structural Health Monitoring of Building Structures. IEEE Systems Journal. DOI:10.1109/JSYST.2015.2481380

[16] A. Cataldo, Benedetto E. D., Cannazza G., Monti G. \& Puzzi E. (2016). TDR-based Monitoring of Rising Damp through the Embedding of Wire-like Sensing Elements in Building Structures. Journal of International Measurement Confederation,DOI: 10.1016/j.measurement.2016.10.044. 